\title{
A Statistical Path Loss Model for Medical Implant Communication Channels
}

\author{
Kamran Sayrafian-Pour, Wen-Bin Yang, John \\ Hagedorn, Judith Terrill \\ Information Technology Laboratory \\ National Institute of Standards and Technology \\ Gaithersburg, Maryland, USA \\ \{ksayrafian, wyang, hagedorn, Judith.terrill\}@nist.gov
}

\author{
Kamya Yekeh Yazdandoost \\ Medical ICT Institute \\ National Institute of Information and Communications Technology \\ New Generation Wireless Communications Research Center \\ Yokosuka, Japan \\ yazdandoost@nict.go.jp
}

\begin{abstract}
Knowledge of the propagation media is a key step toward a successful transceiver design. Such information is typically gathered by conducting physical experiments, measuring and processing the corresponding data to obtain channel characteristics. In case of medical implants, this could be extremely difficult, if not impossible. In this paper, an immersive visualization environment is presented, which is used as a scientific instrument that gives us the ability to observe RF propagation from medical implants inside a human body. This virtual environment allows for more natural interaction between experts with different backgrounds, such as engineering and medical sciences. Here, we show how this platform has been used to determine a statistical path loss model for medical implant communication systems.
\end{abstract}

Keywords - Channel model; Implant Communication system; Immersive visualization system; Body Area Networks

\section{INTRODUCTION}

Body Area Networks which consist of RF-enabled wearable and implantable sensory nodes are poised to be a promising interdisciplinary technology with novel uses in pervasive health information technology. However, numerous challenges including size, cost, energy source, sensing/actuator technology, transceiver design still need to be resolved [3]. Radio-enabled implantable sensor nodes offer a revolutionary set of applications, among which we can point to smart pills for precision drug delivery, glucose monitors, blood pressure sensing system, and eye pressure sensors for glaucoma patients. Some recent advances in microelectronics indicate that the technology to achieve ultra-small and ultra low power devices is completely within reach.

The Federal Communication Commission (FCC) has allocated the frequencies in the $402-405 \mathrm{MHz}$ range to be used for Medical Implant Communication Services (MICS). This is an ultra-low power, unlicensed mobile radio service for transmitting data in support of diagnostic or therapeutic functions associated with implanted medical devices. The MICS permits individuals and medical practitioners to utilize ultra-low power medical implant devices, such as cardiac pacemakers and defibrillators without causing interference to other users of the electromagnetic radio spectrum. Among primary reasons for selecting these frequencies, one can point to better propagation characteristics for medical implants, reasonable sized antennas and worldwide availability.

Knowledge of RF propagation for implantable devices will assist RF engineers to optimize physical layer design and therefore, achieve better performance. Such information is typically gathered by conducting physical experiments, measuring and processing the corresponding data to obtain channel characteristics. In case of medical implants, this could be extremely difficult if at all possible.

In this paper, we use a sophisticated and innovative 3D virtual reality simulation platform to study electromagnetic propagation from medical implants. Communication with an implanted device can be done from any direction inside or outside the body due to various body postures and human motion. Consequently, a true $3 \mathrm{D}$ environment is needed to better capture, visualize and understand RF propagation from/to implants. In the following sections, we describe such a platform and show how it was used to extract a simple statistical path loss model for MICS channels.

The rest of this paper is as follows. Section 2 will describe the immersive 3D platform that we have constructed to study RF propagation from radio implants. Brief description of our approach and the simulated scenarios is provided in Section 3. Then, the statistical path loss model is described in Section 4, and finally concluding remarks and future plans are expressed in Section 5.

\section{A 3D VISUAIZATION SYSTEM FOR MEDICAL IMPLANTS}

Figure 1 shows the block diagram of our simulation system. As observed, the main components of this system include: a three-dimensional human body model, the propagation engine which is a three-dimensional full-wave electromagnetic field simulator (i.e. $\mathrm{HFSS}^{1}$ ), the 3D immersive \& visualization platform, and finally an implantable (or body surface antenna).

\footnotetext{
${ }^{1}$ HFSS is registered trademark of Ansoft Corporation. The HFSS has been used in this research to foster understanding. Such identification does not imply recommendation or endorsement by the National Institute of Standard and Technology, nor does it imply that this product is necessarily the best available for the purpose.
} 
The 3D human body model includes frequency dependent dielectric properties of $300+$ parts in a male human body. These properties are also user-definable if custom changes or modifications are desired. The human body model has a resolution of $2 \mathrm{~mm}$. The HFSS propagation engine enables us to compute a variety of different electromagnetic quantities such as the magnitude of electric and magnetic fields and Specific Absorption Rate (SAR).

The 3D immersive platform as shown in Fig. 2 includes several important components: three orthogonal screens that provide the visual display, the motion tracked stereoscope glasses, and the hand-held motion tracked input device. The screens are large projection video displays that are placed edgeto-edge in a corner configuration. These three screens are used to display a single three-dimensional (3D) stereo scene. The scene is updated based on the position of the user as determined by the motion tracker. This allows the system to present to the user a 3D virtual world within which the user can move and interact with the virtual objects. The main interaction device is a hand-held three button motion-tracked wand with a joystick.

This virtual environment allows for more natural interaction between experts with different backgrounds such as engineering and medical sciences. The user can look at data representations at any scale and position, move through data, change orientation, and control the elements of the virtual world using a variety of interaction techniques including measurement and analysis [2]. All of these capabilities are extremely useful when studying RF propagation to/from medical implants.

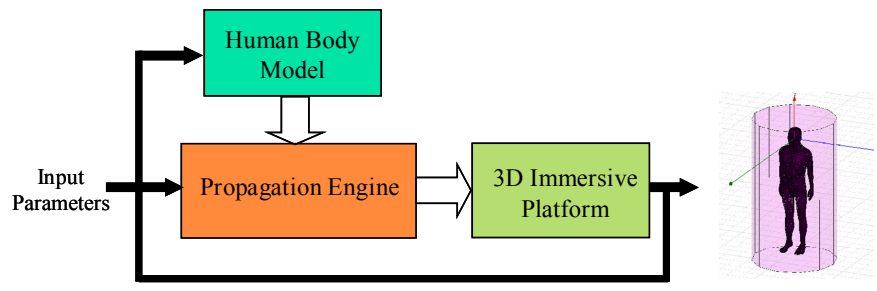

Figure 1. System block diagram

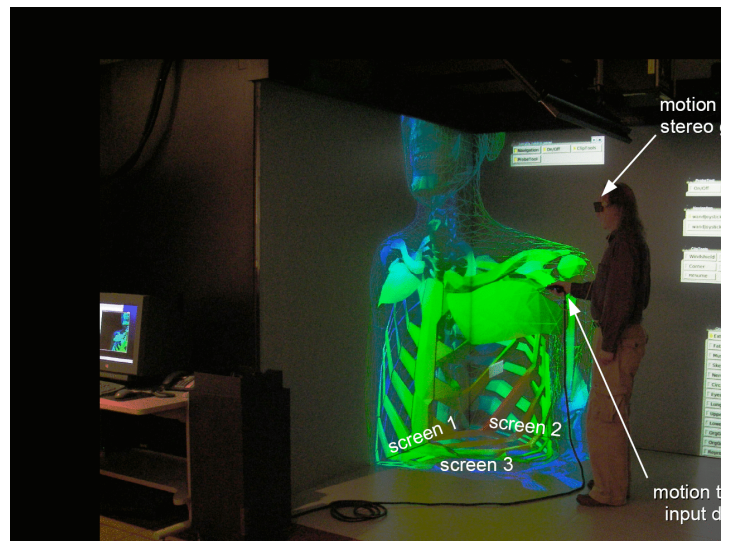

Figure 2. A User in the NIST Immersive Visualization Environment
The final component of our system is the implant antenna. The operating environment for an implant antenna is quite different from the traditional free space communication. Designing an efficient antenna for implantable devices is an essential requirement for reliable MICS operation. The dimension of the antenna must be very small and it should be long term biocompatible [5]. The antenna must also be electrically insulated from the body to avoid possible short circuits.

Figure 3 displays the implant antenna used in our simulations. The antenna is composed of a single metallic layer and is printed on a side of a D51(NTK) substrate with dielectric constant of $\varepsilon_{r}=30$, loss tangent of $\tan \theta=0.000038$, and thickness of $1 \mathrm{~mm}$. A copper of 0.036 $\mathrm{mm}$ thickness has been used as a metallic layer. The dimension of the antenna is $8.2 \times 8.1 \times 1 \mathrm{~mm}$ which is quite appropriate for some medical applications. The metallic layer is covered by RH-5 substrate with dielectric constant of $\varepsilon_{r}=1.0006$, loss tangent of $\tan \theta=0$, and thickness of $1 \mathrm{~mm}$. The simulated return loss of this antenna is shown in Fig. 4. Good impedance matching in the MICS frequency band (i.e. $402-405 \mathrm{MHz}$ ) is observed. Further details on the design and performance of this antenna can be found in $[1,6]$.

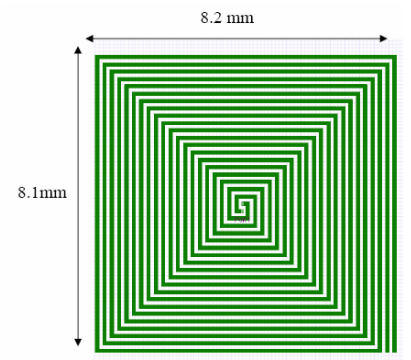

Figure 3. The layout of the implant antenna

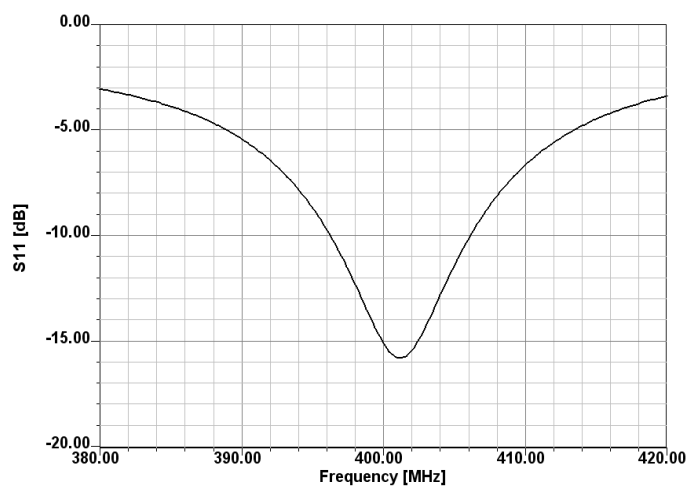

Figure 4. Return loss of the implant antenna

Input parameters to our system include: antenna characteristics, antenna position, antenna orientation, operating frequency, transmit power, resolution, range and the choice of the desired output parameters. The operating frequency in this study has been chosen to be $403.5 \mathrm{MHz}$ which is the mid-point of the MICS frequency band. Resolution of $4 \mathrm{~mm}$ has been selected to run the simulation and a range (i.e. distance) of $50 \mathrm{~cm}$ from the transmitting antenna has been considered. 
Figure 5 displays a sample output image of our system when an antenna is located in the right arm. The color map shows the received signal strength on the surface of the body.

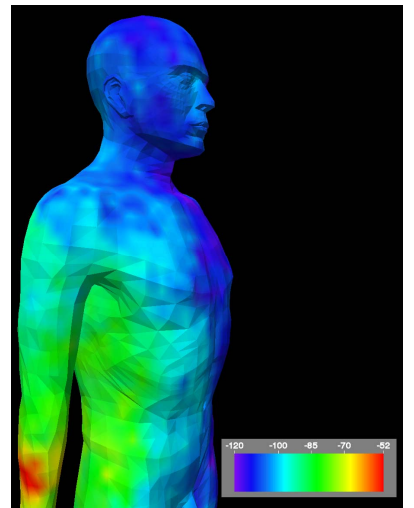

Figure 5. Sample output image of the immersive system representing signal strenght

\section{APPROACH}

Simulations have been performed for four near-surface implants and two deep-tissue implants applications in a typical male body. The near-surface scenarios include applications such as Implantable Cardioverter-Defibrillator (ICD) and Pacemaker (located below the left pectoral muscle), Vagus Nerve Stimulation (Right Neck \& Shoulder) and two Motion Sensor applications located in right hand and right leg. These locations are shown by the white circles in Fig. 6. The deep tissue implant scenarios considers endoscopy capsule applications for upper stomach (95mm below body surface) and lower stomach (118mm below body surface) as highlighted by the red circles in Fig. 6 .

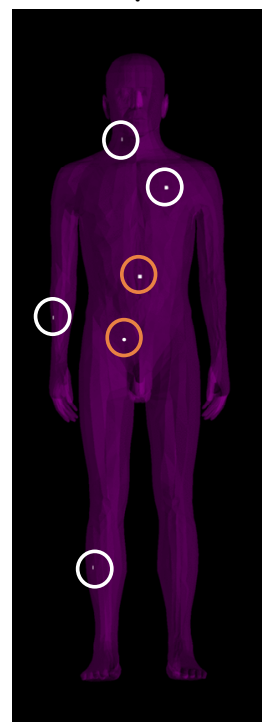

Figure 6. Various transmitter locations for obtaining RSS

For each scenario (i.e. TX location), the received power was calculated for a grid of points within a cylinder area around the body as shown in Fig. 7. Then, the resulting data was partitioned into three sets: in-body to in-body, in-body to body surface, and in-body to out-body propagation sets. The in-body to in-body set includes all of the sample points that completely reside inside the body. Likewise, the in-body to body surface set includes all points that reside within a definable distance (i.e. $2 \mathrm{~mm}, 10 \mathrm{~mm}$, and $20 \mathrm{~mm}$ ) from the body surface; and finally the in-body to out-body propagation set distinguishes all of the points that reside further away from the body surface.

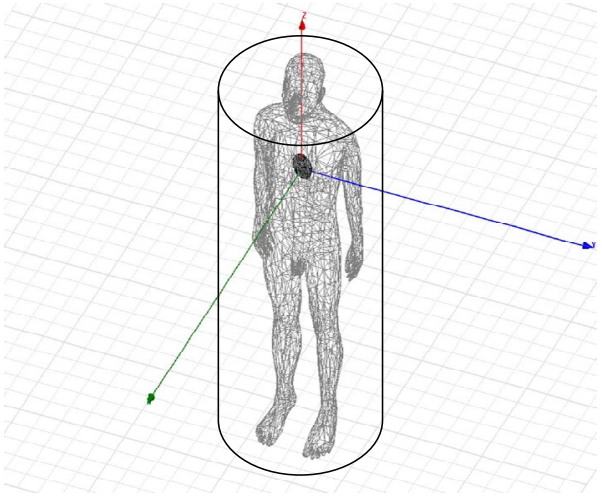

Figure 7. Partitioning the simulation data points

\section{STATISTICAL PATH LOSS MODEL}

In our calculation, path loss at a distance $d$ from the transmitting antenna is defined as:

$$
P L(d)=\frac{G_{R} P_{T}}{P_{R}(d)}
$$

where $P_{T}$ is the transmit power, $P_{R}$ denotes the received power and $G_{R}$ is the receiver antenna gain. Therefore, as defined in above, the path loss would include the transmitter antenna gain. This is usually not the case for channel models corresponding to most wireless systems, but for MICS, the transmitting antenna is considered to be part of the channel [4].

The path loss in $\mathrm{dB}$ at some distance $d$ can be statistically modeled by the following equation:

$$
P L(d)=P L\left(d_{0}\right)+10 n \log _{10}\left(d / d_{0}\right)+S \quad d \geq d_{0}
$$

where $d_{0}$ is the reference distance (i.e. $50 \mathrm{~mm}$ ), and $n$ is the path loss exponent which heavily depends on the environment where RF signal is propagating through. For, example, it is well known that for free space, $n=2$. Human body is an extremely lossy environment; therefore, much higher value for the path loss exponent is expected. $S$ is the random scatter around the mean and represents deviation in $\mathrm{dB}$ caused by different materials (e.g., bone, muscle, etc.) and antenna gain in different directions.

We can model the path loss in two different ways. First method is to find the values for $P L\left(d_{0}\right), n$ and $S$ for the entire data set and only separating the data into implant-to-implant and implant to body surface sets. The second method is to consider separate sets of parameters (i.e. $P L\left(d_{0}\right), n$ and $S$ ) for deep tissue implant versus near-surface implant. Here, we have 
chosen the second method in order to gain some insight into possible differences between these two scenarios.

Figure 8 shows the scatter plot for the path loss as a function of TX-RX separation for deep tissue implant-toimplant scenarios described in the previous section. The mean value of the random path loss has been displayed by a solid line. This is obtained by fitting a least squares linear regression line through the scatter of measured path loss sample points in $\mathrm{dB}$ such that the root mean square deviation of sample points about the regression line is minimized. Random shadowing effects of the channel occur where the TX-RX separation is the same, but have different directions or positions with respect to each other. As shown in Fig. 9, this random variable has a normal distribution with zero mean and standard deviation $\sigma_{s}$ i.e. $S \sim N\left(0, \sigma_{s}^{2}\right)$.

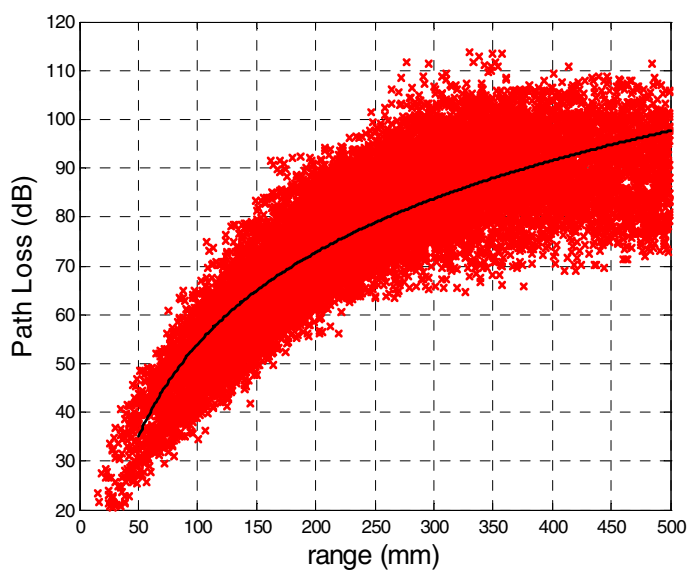

Figure 8. Scatter plot of the path loss versus distance for deep tissue implant to another implant

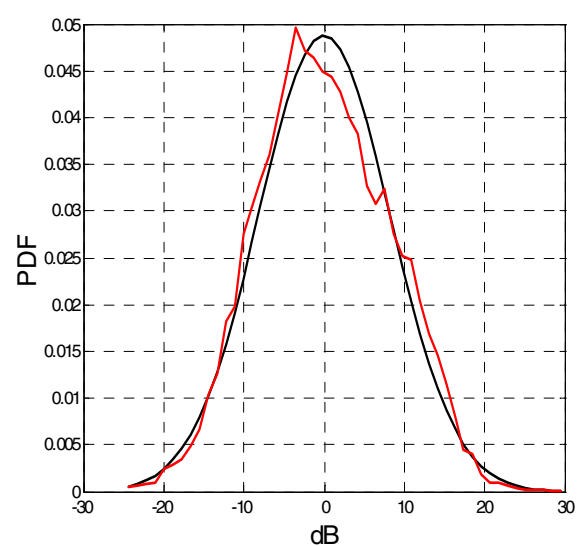

Figure 9. Distribution of the shadow fading for deep tissue implant to another implant

Similarly, Fig. 10 and Fig. 11 represent the path loss scatter plot and probability density function of the shadow fading random variable for the deep tissue implant to body surface scenarios. A distance of up to $20 \mathrm{~mm}$ directly from the body surface has been considered in the definition of the body surface sample points. We also tried $2 \mathrm{~mm}$ and $10 \mathrm{~mm}$ distances and observed that the derived path loss parameters did not vary much. One should keep in mind that layers of clothing could cause additional loss to the signal. Plots corresponding to near surface implant scenarios have been omitted for brevity.

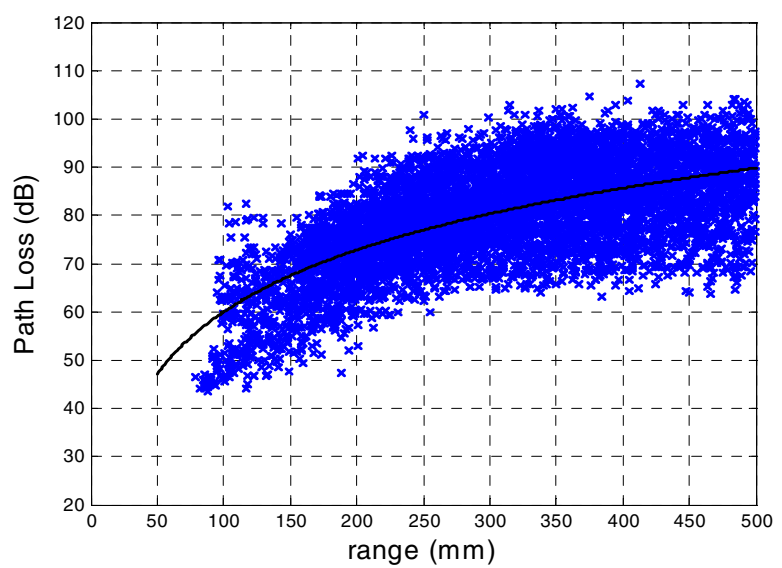

Figure 10. Scatter plot of the path loss versus distance for deep tissue implant to body surface (within $20 \mathrm{~mm}$ of the skin)

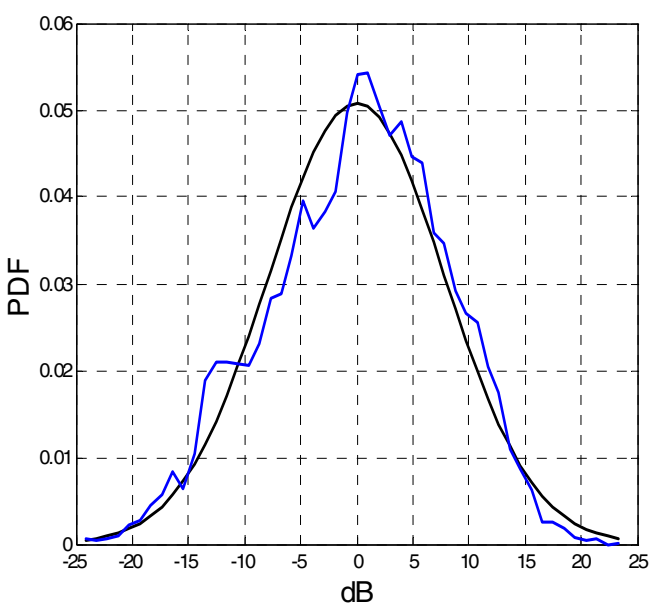

Figure 11. Distribution of the shadow fading for deep tissue implant to body surface (within $20 \mathrm{~mm}$ of the skin)

Table 1 summarizes the extracted parameters for the statistical path loss models decribed above.

\begin{tabular}{|c|c|c||c|}
\hline $\begin{array}{c}\text { Implant to Body } \\
\text { Surface }\end{array}$ & $P L\left(d_{0}\right)(d B)$ & $n$ & $\sigma_{s}(d B)$ \\
\hline Deep Tissue & 47.14 & 4.26 & 7.85 \\
\hline Near Surface & 49.81 & 4.22 & 6.81 \\
\hline
\end{tabular}


(a)

\begin{tabular}{|c|c||c|c|}
\hline $\begin{array}{c}\text { Implant to } \\
\text { Implant }\end{array}$ & $P L\left(d_{0}\right)(d B)$ & $n$ & $\sigma_{s}(d B)$ \\
\hline Deep Tissue & 35.04 & 6.26 & 8.18 \\
\hline Near Surface & 40.94 & 4.99 & 9.05 \\
\hline
\end{tabular}

(b)

Table 1. Parameters for the statistical path loss model: (a) Implant to body surface channel (b) Implant to implant channel

Deriving a channel model for communication between an implant and another node that resides further away from the body is more challenging. In the simple case of an environment where there are no objects or obstacles, a free space path loss can be added to the above models to account for the additional loss that the implant signal will go through once it leaves the body. On the other hand, if there are objects adjacent to the body in the surrounding environment, further loss could occur and the model needs to be adjusted accordingly. With appropriate assumptions this channel could be considered as an aggregate of two concatenated channels: implant to body surface and body surface to the external node channels. Further analysis and data is needed to investigate this issue and obtain the appropriate statistical models.

\section{CONCLUSION}

In this paper, we have constructed an immersive visualization environment to conduct research in order to characterize RF propagation from medical implants. Extensive simulations have been performed to obtain a statistical path loss model for MICS channels. The model is based on four near surface and two deep tissue implant applications in a typical male human body. The authors recognize the fact that the extracted parameters for the statistical model are based on the simulation data; therefore, upon availability of measurement data from physical experiments, the results outlined in this paper should be further validated. However, no such reference data set is currently available. Along this line, efforts are underway to conduct and obtain body surface measurements which can be emulated in the immersive system for cross-verification purposes.

The path loss models obtained in this study have been adopted by the IEEE802.15 task group TG6 on body area networks. The accuracy and applicability of such models requires further validation and investigation by scientists and engineers.
The 3D visualization platform can also be used to study body surface propagation for wearable devices that operate at other frequency bands (e.g. ISM, UWB). It is also an ideal environment to observe and study the creeping wave propagation on the body surface resulting from wearable devices. We are currently studying this phenomenon and further information will be provided in a future publication.

In general, studying a specific medical implant application with custom made antennas is also possible with this platform. More in-depth research on this subject is undoubtedly required to further understand the characteristics of radio frequency propagation from medical implants. The authors hope that the virtual reality environment introduced here would create a flexible platform where efficient collaboration between engineers and medical experts would become possible.

\section{ACKNOWLEDGMENT}

The authors would like to express their gratitude to Nicholas Hirth and Ken Ferreira from Ansoft Corporation for their assistance and support in applying the body model to the 3D immersive system.

We would also like to thank Steven Satterfield of the Scientific Applications and Visualization Group for his assistance in system setup and his ongoing contribution in the development of tools for the NIST immersive visualization system.

\section{REFERENCES}

[1] K.Y.Yazdandoost, R. Kohno, "An Antenna for Medical Implant Communication Systems", Proceedings of the $37^{\text {th }}$ European Microwave Conference, Oct. 2007, Munich, Germany

[2] J. G. Hagedorn, J. P. Dunkers, S.G. Satterfield, A. P. Peskin, J. T. Kelso, J. E. Terrill, "Measurement tools for the immersive visualization environment: Steps toward the virtual laboratory", Journal of research of the National Institute of Standard \& Technology, Vol. 112, Num. 5, Sept.-Oct. 2007

[3] G. Yang, "Body sensor networks", Springer-Verlag London Limited 2006, ISBN 1-84628-272-1

[4] P. S. Hall, Y. Hao, "Antennas and propagation for body centric wireless communications", Artech House, Inc. 2006, ISBN 158053-493-7

[5] C. Polk, E. Postow, "Biological effects of electromagnetic fields", CRC Press, Inc., 1996, ISBN 0-8493-0641-8

[6] K.Y.Yazdandoost, R.Kohno, "Body Implanted Medical Device Communications", IEICE Trans. Comm., Vol. E92-B, No. 2, Feb. 2009 